\title{
Deficiências Nutricionais: Ações Específicas do Setor Saúde para o seu Controle
}

\section{Nutritional Deficiencies: Specific Control Measures by the Health Sector}

\author{
Malaquias Batista Filho ${ }^{1}$ \\ Anete Rissin ${ }^{2}$
}

\section{BATISTA-FILHO, M. \& RISSIN, A. Nutritional Deficiencies: Specific Control Measures by the} Health Sector. Cad. Saúde Públ., Rio de Janeiro, 9 (2): 130-135, Apr/Jun, 1993.

The authors describe and analyze the health sector's role in relation to ways of controlling major endemic nutritional deficiencies by reviewing the theoretical groundwork and establishing possible regression estimates for the various nutritional problems under study. The article is a contribution to the analysis of sectorial alternatives elaborated according to United Nations goals (UNICEF - WHO - FAO) for the year 2000. Among these goals, we stress reduction of the proportion of low birth weight (less than 2500 grams) to less than 10\%; reduction of anemia cases to one-third of present cases; a reduction of $50 \%$ in prevalence of moderate and severe cases of malnutrition and the virtual control of vitamine A and iodine deficiencies.

Key words: Nutritional Disorders; Prevention \& Control; Health Services

\section{INTRODUÇÃO}

As deficiências nutricionais de maior importância epidemiológica - a desnutrição energético-protéica (DEP), as anemias, a hipovitaminose A e o bócio - acham-se estreitamente associadas ao quadro estrutural da pobreza. Em consequiência, sua erradicação completa, definitiva e legítima encontra-se na dependência da própria erradicação dos grandes contrastes econômicos e sociais gerados e mantidos pelo processo de produção e distribuição de bens e serviços.

Impõe-se, portanto, uma vigorosa ação de governo, respaldada pelo consenso da sociedade, como meio de reverter, a médio e longo prazos, o contexto político, econômico e social que condiciona as diferentes manifestações das carências alimentares e nutricionais (Koifman, 1988; Carlson \& Wardlaw, 1990).

A despeito destas considerações, reconhece-se

\footnotetext{
${ }^{1}$ Instituto Materno-Infantil de Pernambuco. Rua dos Coelhos, 300, Boa Vista. Recife, PE, 50070-550, Brasil

${ }^{2}$ Fundação Nacional de Saúde-PE. Avenida Conselheiro Rosa e Silva, 1489, Tamarineira. Recife, PE, 52050-020, Brasil.
}

que o setor saúde pode, a curto e médio prazos, contribuir para uma efetiva redução do quadro de deficiências nutricionais utilizando uma série de instrumentos de comprovada eficácia. É a lógica das intervenções próprias do setor saúde que se pretende analisar neste trabalho, estabelecendo os fundamentos e as projeções de algumas medidas que podem e devem ser adotadas como estratégias específicas para o controle de processos carenciais.

\section{FUNDAMENTAÇÃO}

Para se compreender bem o papel do setor saúde, de forma isolada ou como componente de uma proposta integrada de combate aos problemas carenciais, torna-se necessário revisar os conceitos básicos do modelo causal das deficiências nutricionais, integrando elementos fisiopatológicos e suas implicações epidemiológicas (Béghin \& Dujardim, 1984).

1. O estado de nutrição expressa a disponibilidade e o aproveitamento metabólico de energia e nutrientes a nível de células e tecidos. Trata-se de uma condição delineada 
por duas grandes vertentes: o consumo de alimentos, por um lado, e a sua utilização biológica, por outro (Béghin, 1990).

2. A vertente do consumo alimentar está constituída por fatores culturais, que definem, através de hábitos, os alimentos preferenciais para o consumo familiar e individual, e, ainda, por fatores econômicos, que estabelecem, em última instância, a capacidade de compra e/ou de produção própria da família.

Entende-se, até por consenso vulgar, que, nesta área, o setor saúde tem pouca contribuição a oferecer. Duas possibilidades - de alcance imediato um tanto limitado — podem ser vislumbradas: a) orientação educativa, no sentido de modificar hábitos de consumo, no que se refere à escolha, combinação e preparação dos alimentos; b) distribuição direta de suplementos alimentares, como o leite, formulados ou nutrientes sob a forma de fármacos (vitaminas, sais minerais).

3. A vertente mais apropriada para as ações do setor saúde refere-se ao monitoramento de fatores que dizem respeito ao aproveitamento biológico de energia ou nutrientes.

As doenças alteram significativamente o padrão fisiológico de aproveitamento dos alimentos, na medida em que, por sua natureza (ação patogênica e duração), podem comprometer, de forma marcante:

a) o consumo alimentar, por redução do apetite, ocorrência de vômitos ou conjugação destes dois efeitos. É um evento comum nas doenças infecciosas;

b) a digestão, por aceleração do trânsito gastrintestinal e alteração do padrão enzimático da digestão. As diarréias tipificam este item;

c) a absorção de nutrientes (digestão incompleta, trânsito acelerado, lesões de mucosa, inversão do fluxo hidroetrolítico), o que pode ser bem ilustrado nos casos de diarréia;

d) as necessidades nutricionais, exageradas por elevação de temperatura, aumento do catabolismo protéico, de vitaminas e minerais. Os processos febris são exemplos bem concretos desta situação. e) Finalmente, as doenças podem contribuir para aumentar as perdas de nutrientes, por via intestinal, urinária ou cutânea.

A simples menção destes eventos adversos, comprometendo, de forma significativa, as diferentes etapas do processo fisiológico da nutrição, presta-se para uma compreensão imediata da importância das doenças (sobretudo as infecções) na gênese das deficiências nutritivas.

Afortunadamente, já se logrou um avanço considerável (e, às vezes, até definitivo) no controle das doenças infecciosas, mediante recursos simples de promoção, proteção específica e recuperação da saúde. $O$ fato de se dispor de tecnologias simples e de baixo custo, como o tratamento público ou domiciliar da água, o controle de vetores, as vacinações e a terapia de reidratação oral, estabelece uma perspectiva considerável para o resgate dos efeitos adversos das doenças sobre o estado nutricional (Kennedy \& Alderman, 1983; Berg, 1984).

Tal constatação é de uma importância estratégica fundamental, deslocando o campo de atuação do setor saúde da ênfase alimentar, dominante até meados da última década, para o espaço próprio do controle de doenças e vigilância do crescimento, que passam a representar o enfoque hegemônico e convencional do setor saúde.

\section{GRUPOS ALVOS}

Em princípio, toda a população acha-se exposta aos riscos nutricionais. No entanto, em termos de saúde coletiva, somente alguns segmentos biológicos e sociais apresentam, de fato, um interesse marcante, em razão da magnitude, dos agravos (mortes, incapacitações temporárias ou permanentes) e das possibilidades concretas de se beneficiar com ações específicas de saúde. É o caso de gestantes, lactentes e pré-escolares.

Estas características facilitam a identificação de grupos alvos, a seleção de problemas e a aplicação de medidas de intervenção oportunas e pertinentes, utilizando-se critérios de risco e variáveis estratégicas para cada situação genérica. 


\section{PROBLEMAS E INTERVENÇÕES}

\section{Gestantes}

Em relação às gestantes, dois problemas carenciais são de importância prioritária: a desnutrição energético-protéica (DEP) e as anemias (Ebrahim, 1983; Worthington-Roberts \& Vermeersch, 1986; Figueira et al., 1991).

A desnutrição energético-protéica (DEP) pode ser facilmente presumida pela tomada de peso e altura e avaliação do peso gestacional, mediante o método de Rosso (1985). No meio rural, a simples aplicação de uma fita no terço médio do braço pode estabelecer uma boa triagem entre mães sob risco e gestantes "normais" (Barros, 1990).

$\mathrm{O}$ uso de suplementos alimentares (400 cal/dia) no último trimeste de gestação, a um custo baixíssimo, que pode ser bancado pelo poder público em casos de reconhecida necessidade, seria suficiente para corrigir $60 \%$ ou mais dessas situações e suas conseqüências (Lechtig et al., 1972). O diagnóstico e tratamento de processos infecciosos agregados, a redução do consumo de cigarros e o controle de intercorrências outras, mediante exames pré-natais, constituem intervenções que devem ser associadas à vigilância do estado nutricional da gestante.

O problema das anemias pode ser convenientemente enfrentado com o uso profilático ou curativo de sais de ferro (Ebrahim, 1983). Mais de 3/4 das anemias podem ser proveitosamente trabalhados com a terapia medicamentosa, aplicada de forma perseverante (3 a 4 meses), com doses bem-toleradas e bem-distribuídas. $\mathrm{O}$ custo da intervenção é praticamente desprezível. São medidas complementares: orientação dietética, planejamento familiar, bem como condições e práticas sanitárias que permitam reduzir as agressões infecciosas.

\section{Crianças}

Três problemas carenciais são de importância fundamental em menores de 5 anos: a desnutrição energético-protéica (DEP), as anemias e a deficiência de vitamina A.

A DEP se manifesta através do baixo peso ao nascer (menos de 2.500 gr) e do déficit de crescimento (altura/idade, peso/idade e peso/altura) nos primeiros anos de vida.

A prevenção do baixo peso ao nascer se faz mediante cuidados pré-natais, já devidamente assinalados no texto referente à nutrição materna. Por outro lado, a redução da prevalência do baixo pelo ao nascer representa a medida inicial mais válida para a própria prevenção da desnutrição nos primeiros meses e anos de vida, já que o déficit de crescimento está fortemente associado ao baixo peso de nascimento (Victora et al., 1988; Pires de Souza, 1991).

Em relação aos 6 primeiros meses de vida, é essencial o estímulo ao aleitamento materno, que, nas famílias de baixa renda, se comporta como a variável mais importante para reduzir a mortalidade e a desnutrição. Mais do que seu valor nutritivo propriamente dito, o leite materno representa uma excelente alternativa para se diminuir a ocorrência de doenças, reduzindo, desta forma, o risco de desnutrição secundária aos agravos infecciosos (Unicef, 1991). O estímulo ao aleitamento materno pode ser feito no pré-natal, na assistência ao parto e puerpério (alojamento conjunto), nos cuidados assistenciais com a criança e através da utilização dos meios de comunicação de massa.

Após os 6 primeiros meses de vida, torna-se relevante o acompanhamento das mudanças qualitativas e quantitativas nas práticas alimentares das crianças. Em muitos casos, pode se tornar necessária a suplementação alimentar através dos próprios serviços de saúde. O leite é o alimento eletivo nestas condições.

Tanto nas crianças menores de 6 meses como nas de idades maiores, é indispensável, pelas razões já enunciadas no item de fundamentação, adotar as medidas possíveis, objetivando a prevenção de doenças como as diarréias, infecções respiratórias e imunopreveníveis ou, em última instância, o diagnóstico e tratamento eficaz destas intercorrências, de modo a minimizar seu impacto sobre o estado de nutrição. A estratégia de atenção primária à saúde aplicada de forma eficiente (agentes devidamente treinados e supervisionados, atuando sob estreita articulação com os níveis de atenção secundária) pode ter um papel relevante no desempenho destas ações, com surpreendente impacto 
sobre o estado de nutrição, como se demonstra com a experiência da Costa Rica e do norte da Argentina (Arias, 1988).

Um instrumento axial da assistência deve ser o acompanhamento constante do crescimento e desenvolvimento da criança, como pólos de gravitação de todo o programa de atenção integral à saúde da criança. As doenças e a desnutrição se refletem, de forma sensível, no ritmo de crescimento e, numa instância subseqüente, no processo de desenvolvimento.

Outra situação a ser mantida sob controle são as anemias, que ocorrem com frequiência de 30 até $70 \%$ em crianças de 6 meses a dois anos de idade (Steckel, 1984).

A prevenção do baixo peso ao nascer e da anemia materna no curso da gestação são duas medidas que reduzem, de modo substancial, o risco de déficit de hemoglobina nos primeiros meses. O aleitamento materno até os 6 meses de vida é outra condição importante para se evitar o problema. Como se observa, estas intervenções já foram mencionadas em outras instâncias, ressaltando-se aqui seus papéis no que se refere à hemoglobina circulante.

Como ainda não se dispõe de uma boa experiência neste sentido, considera-se conveniente a administração, após os 6 meses de vida, de sais de ferro em suspensão na base diária de 6 $\mathrm{mg}$, representando uma alternativa farmacológica de se prevenir a anemia ferropriva. É importante, ademais, orientar as mães no sentido de utilizar alimentos ricos em ferro - ovos, carnes, vísceras - no processo de transição alimentar da criança.

O controle de infecções e, sobretudo, de enteroparasitoses completaria o elenco de medidas de uma rotina mínima para o monitoramento das anemias.

Por último, deve-se referir o problema da deficiência de vitamina A em crianças.

$\mathrm{O}$ aleitamento materno é a medida eletiva para a prevenção da deficiência até os 6 meses de vida. Em caráter complementar e substitutivo, deve-se investir na adoção do leite integral, já que as fórmulas lácteas desengorduradas são habitualmente privadas ou incompletas, sob o ponto de vista de vitamina $\mathrm{A}$.

Como uma medida a curto prazo, de baixo custo e elevada eficácia, a estratégia mais indicada seria a aplicação sistemática de vitami- na A duas vezes por ano, aproveitando-se os chamados "dias nacionais de vacinação". A administração de doses maciças de vitamina A (200.000 U. I. de cada vez, duas vezes por ano) reduziria em mais de $90 \%$ o risco de instalação de quadros clínicos ou subclínicos desta deficiência. Dispõe-se de evidências, na Indonésia, de que o controle desta deficiência (pouco valorizada nas práticas de saúde) poderia reduzir em até $30 \%$ a mortalidade em crianças menores de 5 anos.

Mais uma vez, o controle de doenças infecciosas e parasitárias (com ênfase na giardíase) constitui um instrumento auxiliar estratégico para a prevenção da deficiência.

É interessante consultar o manual de $\mathrm{MacAu}$ life et al. (1991) sobre o assunto.

\section{Outras Carências}

Admite-se que 2/3 dos casos de cárie dental podem ser atribuídos à deficiência específica de flúor. A fluoretação das águas de abastecimento público e métodos alternativos (aplicação tópica de soluções fluoretadas) podem reduzir a incidência de cárie, que representa o principal fator de demanda nos serviços odontológicos.

$\mathrm{O}$ bócio iodoprivo, agora praticamente sob controle no Brasil, deve ser vigiado, mediante o monitoramento da iodatação do sal de consumo humano nos laboratórios de saúde pública.

É possível que, dentro de poucos anos, a deficiência de zinco e de vitaminas do complexo B (principalmente a tiamina e a riboflavina) venha a ser considerada um problema de interesse sanitário.

\section{PERSPECTIVAS}

Existe um compromisso internacional (Conferência de Cúpula de Nova Iorque, 1990) no sentido de se alcançar, até o ano 2000, em todos os países do mundo, algumas metas específicas de nutrição. Figuram neste elenco a redução do baixo peso ao nascer (abaixo de 2.500 gramas) para menos de $10 \%$, a diminuição das anemias em 1/3, o declínio em 50\% das formas moderadas e graves da DEP, e, por fim, o controle virtual do bócio iodoprivo e da 
hipovitaminose A nas áreas de reconhecida endemicidade (Unicef, 1990). A situação encontrada em 1990 constitui a referência temporal para se estimar tais metas.

$\mathrm{Na}$ quase totalidade das situações, excetuando-se as conjunturas alimentares críticas que prevalecem em determinadas regiões (como nos países do Sahel, na zona semiárida do Nordeste e nas áreas montanhosas do istmo centro-americano), é possível que apenas acionando medidas de saúde, mantendo-se constantes as condições sócio-econômicas básicas, seja provável alcançar, com relativa segurança, as metas propostas.

O know-how oferecido por projetos experimentais e, mais importante, os resultados obtidos em escala nacional (Chile, Costa Rica) ou regional (norte da Argentina) oferecem embasamento empírico para se fazer as projeções aqui estabelecidas. No Brasil, a experiência do estado do Ceará (1987-1990) também é ilustrativa de consistência destas estimativas (Unicef/Socep/Hope, 1990).

Conjugadas com outras medidas de atenção integral à saúde da mulher e da criança, a adoção sistemática destas ações poderia resultar, nos próximos 6 a 8 anos, numa reversão de situações, a qual pode ser, tentativamente, assim delineada:

1. Redução da mortalidade infantil em 50\%;

2. Decréscimo entre 50 e $60 \%$ na prevalência de formas moderadas e graves de desnutrição;

3. Diminuição de pelo menos $60 \%$ na freqüência de anemias em crianças e gestantes;

4. Redução em $90 \%$ dos riscos de instalação de quadros de deficiência de vitamina A.

Estes resultados poderiam, inclusive, ser melhorados, na dependência de políticas macroeconômicas bem-sucedidas, com aumento da renda, do nível de empregos e salários, abastecimento e preços. No entanto, o que se pretende ressaltar é que, por si, um programa explícito de ações de saúde poderia, num curto espaço de tempo, contribuir para um declínio substancial no quadro de indicadores que descrevem o perfil nosográfico das grandes endemias carenciais.

\section{RESUMO}

BATISTA-FILHO, M. \& RISSIN, A.

Deficiências Nutricionais: Ações Específicas do Setor Saúde para o seu Controle. Cad. Saúde Públ., Rio de Janeiro, 9 (2): 130-135, abr/jun, 1993.

Os autores descrevem e analisam o papel do setor saúde nos programas de controle das principaiss endemias carenciais, revisando os fundamentos teóricos e estabelecendo possíveis estimativas de reversão para os diversos problemas nutricionais considerados. $\mathrm{O}$ artigo representa uma contribuição para a análise das alternativas setoriais elaboradas em função das metas da Organização das Nações Unidas (Unicef — OMS — FAO) para o ano 2000, entre as quais figuram a redução do baixo peso ao nascer (abaixo de 2.500 gramas) para menos de $10 \%$, a diminuição em $1 / 3$ das anemias, o decréscimo de 50 na prevalência de desnutrições moderada e grave em crianças, e o controle virtual da hipovitaminose A e do bócio iodoprivo.

Palavras-Chave: Distúrbios Nutricionais; Prevenção e Controle; Serviços de Saúde

\section{REFERÊNCIAS BIBLIOGRÁFICAS}

ARIAS, C. V., 1989. El programa de nutrición de Costa Rica. In: Vigilância Alimentaria y Nutricional en las Américas (OPAS), pp. 135-137, Washington: Organización Panamericana de la Salud. (Publicación Científica 516)

ARIAS, L. M.; LOBO, C.; MATEO, S. A. \& BUSTOS, N., 1989. Vigilancia alimentaria y nutricional en la Província de Salta, Argentina. In: Vigilância Alimentaria y Nutricional en las A méricas (OPAS), pp. 111-115, Washington: Organización Panamericana de la Salud. (Publicación Científica 516)

BARROS, L. M., 1990. A Circunferência Braquial e a Curva de Ganho de Peso Ponderal como M étodos de Avaliação do Estado Nutricional da Gestante. Tese de Mestrado, Recife: Departamento de Nutrição, Universidade Federal de Pernambuco.

BÉGHIN, I., 1990. Abordagem causal em nutrição. In: Nutrição, Saúde \& Comunidade (E. L. C. Gouveia, org.), pp. 181-193, Rio de Janeiro: Revinter. 
BERG, A., 1984. LoS Malnutridos: Examen de Políticas. Washington: BIRD. (Série sobre la Pobreza y las Necesidades Básicas)

CARLSON, B. A. \& WARPLAW, T. M., 1990. A Global, Regional and Country Assessment of Child Malnutrition. New York: Fundo das Nações Unidas para a Infância e Adolescência (Unicef Staff Working Papers, 7)

EBRAHIM, G. J., 1983. Nutrition in Mother and Child Health. London: Macmillan. 1983. Nutritional anaemias. In: Nutrition in M other and Child Health (G. J. Ebrahim, org.), p. 167-181, London: Macmillan.

FIGUEIRA, F; BATISTA-FILHO, M.; ARRUDA, I. G. K. \& SANTOS, L. C., 1991. Alimentação e nutrição da gestante e da nutriz. In: Nutrição da Criança (J. Wehba, org.), pp. 273-294, São Paulo: BYK.

KENNEDY, E. T. \& ALDERMAN, H. H., 1987. Comparative Analysis of Nutritional Effectiveness of Food Subsidies and Other Food-related Interventions. Washington: International Food Policy Research Institute, joint WHO-UNICEF Nutrition.

KOIFMAN, S., 1988. Crise econômica e desnutrição. O caso do Rio de Janeiro, Brasil, 1959-1986. In: Crise e Infância no Brasil (J. P. Chahad \& R. Cervini, org.), pp. 335-358, São Paulo: Fundo das Nações Unidas para a Infância e Adolescência/Instituto de Pesquisas Econômicas/Universidade de São Paulo.

LECHTING, A. \& KLEIN, R. E., 1981. Nutrición materna y crecimiento fetal. In: Biologia y Ecologia Fetal (R. Maciá, org.), pp. 123-140, Barcelona: Salvat Editora.

MCAULIFFE, J.; SANTOS, L. M. P.; DINIZ, A. S.; BATISTA-FILHO, M. \& BARBOSA, R. C. C., 1991. Deficiências de Vitamina A e Estratégias para seu Controle. Fortaleza: Project Hope.

MONTEIRO, C. A.; BENÍCIO, H. M. H. D’A.; IUNES, R.; GOUVEIA, N. C.; TADDEI, J. A. A. C. \& CARDOSO, M. A. A., 1992. O estado nutricional de crianças brasileiras: a trajetória de 1975 a 1989. In: Perfil Estatístico de Crianças e Mães no Brasil (Fundo das Nações Unidas para a Infância e Adolescência/Instituto Nacional de Alimentação e Nutrição), pp. 19-42, Rio de Janeiro: Unicef.
PIRES DE SOUSA, F. J. 1992. Pobreza, Desnutrição e Mortalidade Infantil. Condicionantes Sócio-Econômicos. Fortaleza: Fundo das Nações Unidas para a Infância e Adolescência.

ROSSO, P., 1985. A new chart to monitor weight gain during pregnancy. The A merican Journal of Clinical Nutrition, 41: 644-652.

STEKEL, A., 1984. Iron Deficiency in Infancy and Childhood. Vevey: Nestlé Nutrition.

UNICEF (Fundo das Nações Unidas para a Infância e Adolescência), 1990. Estratégia para Melhorar a Nutrição de Crianças e Mulheres nos Países em Desenvolvimento. New York: Unicef. , 1991. Cumprindo a Promessa. Situação Mundial da Infância. New York: Unicef.

UNICEF (Fundo das Nações Unidas para a Infância e Adolescência); OMS (Organização Mundial de Saúde); UNESCO (Organização das Nações para a Educação, Ciência e Cultura), 1991. Medidas Vitais. Um Desafio de Comunicação. Brasília: UNICEF/OMS/UNESCO.

UNICEF (Fundo das Nações Unidas para a Infância e Adolescência); SOCEP; SSE/CE (Secretaria de Saúde do Estado do Ceará); HOPE, 1990. II Pesquisa de Saúde M aterno-infantil no Ceará. Comparação de Dois Estudos de Abrangência Estadual: 1987 - 1990. Fortaleza: Unicef.

VICTORA, C.; BARROS, C. F. \& VAUGHAM, J. P., 1998. Epidemiologia da Desigualdade. São Paulo: Hucitec.

WORTHINGTON-ROBERTS, B. S. \& VERMEERSCH, J., 1986. Nutrição na Gravidez e na Lactação. $3^{\text {a }}$ ed., Rio de Janeiro: Interamericana. 